\title{
Photodestruction of Diatomic Molecular Ions: Laboratory and Astrophysical Application
}

\author{
Vladimir A. Srećković ${ }^{1, * \mathbb{D}}$, Ljubinko M. Ignjatović ${ }^{1}$ and Milan S. Dimitrijević $^{2,3}$ (D) \\ 1 Institute of Physics Belgrade, Belgrade University, Pregrevica 118, 11080 Belgrade, Serbia; ljuba@ipb.ac.rs \\ 2 Astronomical Observatory, Volgina 7, 11060 Belgrade, Serbia; mdimitrijevic@aob.rs \\ 3 Observatoire de Paris, PSL Université, CNRS, Sorbonne Université, F-92195 Meudon, France \\ * Correspondence: vlada@ipb.ac.rs
}

Citation: Srećković, V.A.; Ignjatović, L.M.; Dimitrijević, M.S. Photodestruction of Diatomic Molecular Ions: Laboratory and Astrophysical Application. Molecules 2021, 26, 151. https://doi.org/ 10.3390/molecules26010151

Received: 14 December 2020 Accepted: 28 December 2020 Published: 31 December 2020

Publisher's Note: MDPI stays neutral with regard to jurisdictional claims in published maps and institutional affiliations.

Copyright: (C) 2020 by the authors. Licensee MDPI, Basel, Switzerland. This article is an open access article distributed under the terms and conditions of the Creative Commons Attribution (CC BY) license (https: / / creativecommons.org/ licenses/by/4.0/).
Abstract: In this work, the processes of photodissociation of some diatomic molecular ions are investigated. The partial photodissociation cross-sections for the individual rovibrational states of the diatomic molecular ions, which involves alkali metals, as well as corresponding data on molecular species and molecular state characterizations, are calculated. Also, the average cross-section and the corresponding spectral absorption rate coefficients for those small molecules are presented in tabulated form as a function of wavelengths and temperatures. The presented results can be of interest for laboratory plasmas as well as for the research of chemistry of different stellar objects with various astrophysical plasmas.

Keywords: photodissociation; diatomic molecules; plasma diagnostics; astrophysical processes; radiative processes; molecular spectroscopy; planetary chemistry; planetary geochemistry; atomic and molecular data

\section{Introduction}

Advances in the research of ultra-low-temperature gases have prompted a new interest in alkali-dimer cations (see e.g., [1]). Recently, potential energy curves of some diatomic molecules have been investigated with a special focus both on neutral and ionized alkali-dimer systems [2-4]. Calculations of the potential energy curves for molecules considered here have already been published: $\mathrm{LiH}^{+}$and $\mathrm{NaH}^{+}$in [5], $\mathrm{Li}_{2}^{+}$and $\mathrm{Na}_{2}^{+}$in [6] and for $\mathrm{LiNa}^{+}$in [7]. These data are given in the Supplementary Materials. This particular concern is generally due to the recent development in cold collision dynamics involving alkali diatomic systems.

From [8-10] and from investigation in existing literature about the solar photospheric spectra, the absorption spectra of planetary atmospheres and the chemical composition of dust, it follows that alkali metals take part in the composition of cosmic objects [11,12]. It has been discovered and concluded that important characteristics of the brown dwarfs and extrasolar planets spectra might be attributed to resonance lines of alkali metal atoms broadened by collisions with ambient helium atoms and hydrogen molecules (see e.g., [13,14]). Alkali metal resonance lines shape the optical spectrum from the UV to the near-IR spectral range $[13,15]$.

Most alkali hydride ionic and neutral molecules are very useful in understanding the formation and the evolution of the Molecular Universe [16]. Even though they are involved in various astrophysical and astrochemical processes as radiative transfer or dissociative recombination for instance [17], their spectroscopy is practically unknown both theoretically and experimentally, in particular in the case of molecular ions [18]. The charge transfer in collision of alkali atoms with protons affects the ionization balance in the atmospheres of various environments such as planets, dwarf stars, and the interstellar medium, and is of interest in the area of plasma fusion as a method of energetic neutral beam injection in fusion reactors [19]. Also, one can note the current importance of investigation of optical 
properties of various small molecules (see e.g., [20-24]) and corresponding atomic and molecular (A \& M) data.

In this paper, we will focus on the investigation of photodestruction of the following species: $\mathrm{LiH}^{+}, \mathrm{NaH}^{+}, \mathrm{Li}_{2}^{+}, \mathrm{Na}_{2}^{+}$and $\mathrm{LiNa}^{+}$. Here we will examine the significance of the processes of molecular-ion photodissociation in symmetric case:

$$
h v+M_{2}^{+} \Longrightarrow M+M^{+},
$$

where $M$ and $M^{+}$are alkali atom and ion in their ground states, and $M_{2}^{+}$is molecularion in the ground electronic state. Such processes are photodissociation of $\mathrm{Li}_{2}^{+}, \mathrm{Na}_{2}^{+}$ but also $\mathrm{LiNa}^{+}$ions, keeping in mind a small difference between the ionization energies of $\mathrm{Li}$ and $\mathrm{Na}$ atoms. Also, we will examine the non-symmetric case for the processes of photodissociation:

$$
h v+M X^{+} \Longrightarrow X^{+}+M,
$$

where $M$ is an atom whose ionization potential is less than the corresponding value for atom $\mathrm{X} . \mathrm{MX}^{+}$is also molecular-ion in the ground electronic state. The photodissociation of $\mathrm{HLi}^{+}$and $\mathrm{HNa}^{+}$ions is just an example of strongly non-symmetric processes, keeping in mind that the ionization energy of hydrogen is much higher than the ionization energy of $\mathrm{Li}$ and $\mathrm{Na}$ atoms. Processes $(1,2)$ are schematically shown in Figure 1 by solid arrows. By absorbing photons of the same energy molecular-ion, $\mathrm{MX}^{+}$can pass into one of the higher excited electronic states (dashed arrow) but the probability of these transitions is small compared to the probability of resonant processes $(1,2)$. That is why the influence of the processes represented by the dashed arrow in Figure 1 will not be considered in this paper.

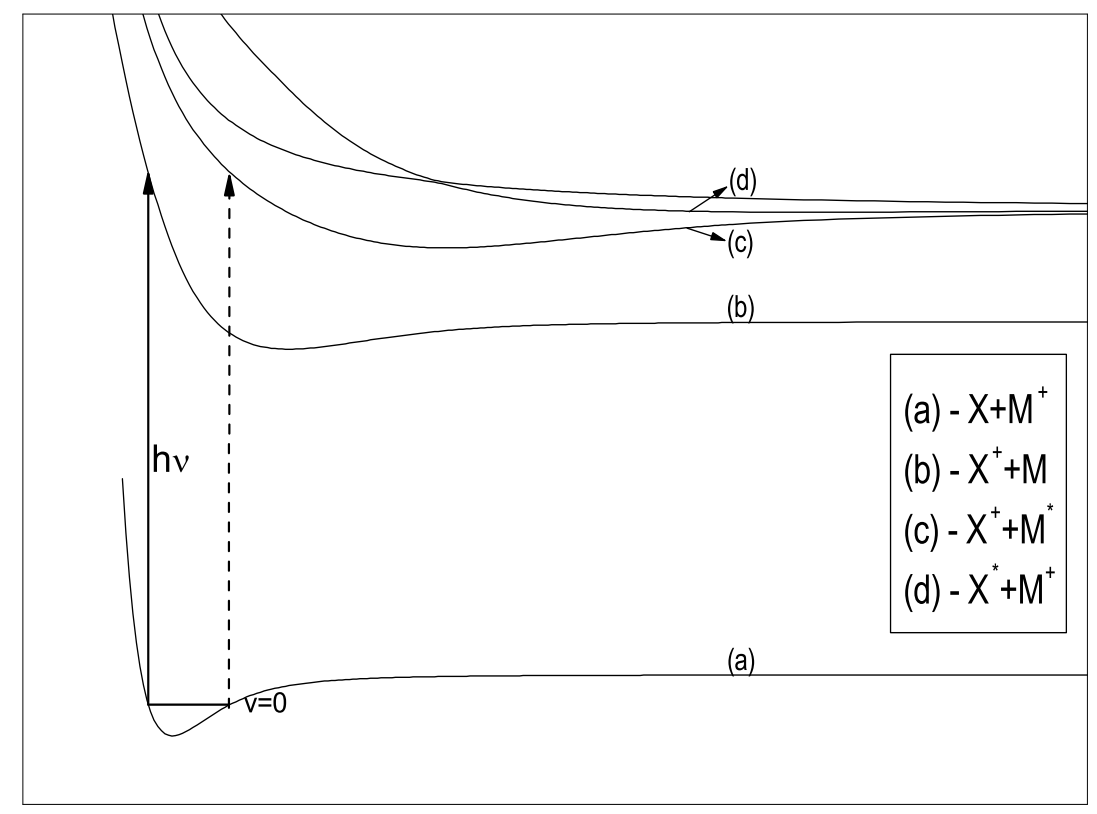

Figure 1. $X$ and $M$ denote atoms in the ground state, $X^{*}$ and $M^{*}$-atoms in the first excited state and $\mathrm{X}^{+}$and $\mathrm{M}^{+}$-atomic ions. Solid arrow: The molecular-ion $\mathrm{MX}^{+}$in the ground state (a) absorbs the photon and passes into the first excited electronic state (b) which decays into the ion $X^{+}$and the $M$ atom in the ground state. Dashed arrow: Molecular-ion $M X^{+}$absorbs a photon and passes into one of the higher electronic states $(\mathbf{c}, \mathbf{d})$ and decays so that one of the atoms remains in the excited state.

We determine theoretically calculated partial photodissociation cross-sections for the individual rovibrational states of the diatomic molecular ions $\mathrm{LiH}^{+}, \mathrm{NaH}^{+}, \mathrm{Li}_{2}^{+}, \mathrm{Na}_{2}^{+}$ and $\mathrm{LiNa}^{+}$as well as the corresponding data on molecular species and molecular state characterizations (rovibrational energy states, etc.). The data are obtained for $\mathrm{Li}_{2}^{+}, \mathrm{Na}_{2}^{+}$ and $\mathrm{LiNa}^{+}$, for $500 \mathrm{~K} \leq T \leq 5000 \mathrm{~K}$ and $400 \mathrm{~nm} \leq \lambda \leq 1000 \mathrm{~nm}$ (for modeling laboratory 
plasmas and geo-cosmic weakly ionized plasma) and for $\mathrm{LiH}^{+}, \mathrm{NaH}^{+}$for extended temperature range $100 \mathrm{~K} \leq T \leq 20,000 \mathrm{~K}$ and wavelength range $60 \mathrm{~nm} \leq \lambda \leq 160 \mathrm{~nm}$ (for additional astro usage in modeling Solar photosphere, atmosphere above sunspots and Lithium-Rich Stars).

Also, the average cross-section and the corresponding spectral absorption rate coefficients for the photodissociation of the mentioned molecular ions are obtained for a wide region of wavelengths and temperatures. The presented results here are ready for further use with a particular emphasis on the applications for astro-plasma research and low-temperature laboratory plasma research [10,13,25-27].

The paper is structured as follows. In Section 3 we describe the methods of calculation and calculated quantities, in Section 2 we present the results of the calculation and discussed results and in Section 4 the conclusions are presented with further directions of research.

\section{Results and Discussion}

The partial photodissociation cross-sections for the individual rovibrational states of the diatomic molecular ions which involve alkali metals are calculated. Also, the average cross-section and the corresponding spectral absorption rate coefficients for those small molecules $\mathrm{LiH}^{+}, \mathrm{NaH}^{+}, \mathrm{Li}_{2}^{+}, \mathrm{Na}_{2}^{+}$and $\mathrm{LiNa}^{+}$are obtained for a wide region of wavelengths and temperatures. The data are obtained for $\mathrm{Li}_{2}^{+}, \mathrm{Na}_{2}^{+}$and $\mathrm{LiNa}^{+}$, for $500 \mathrm{~K} \leq T \leq 5000 \mathrm{~K}$ and $400 \mathrm{~nm} \leq \lambda \leq 1000 \mathrm{~nm}$ (for modeling laboratory plasmas and geo-cosmic weakly ionized plasma) and for $\mathrm{LiH}^{+}, \mathrm{NaH}^{+}$for extended temperature range $100 \mathrm{~K} \leq T \leq 20,000 \mathrm{~K}$ and wavelength range $60 \mathrm{~nm} \leq \lambda \leq 160 \mathrm{~nm}$ (for additional astro usage in modeling Solar photosphere, atmosphere above sunspots and Lithium-Rich Stars). The results are presented in this section by figures and table and in Supplementary Material.

\subsection{Cross-Sections}

In Figures $2 \mathrm{a}, \mathrm{b}$ and $3 \mathrm{a}, \mathrm{b}$ we present a surface plot of the averaged cross-section $\sigma(\lambda, T)$ for photodissociation of the $\mathrm{LiH}^{+}, \mathrm{NaH}^{+}, \mathrm{Li}_{2}^{+}$and $\mathrm{LiNa}^{+}$molecular ions as a function of $\lambda$ for a wide range of temperatures $T$, which are relevant for modeling laboratory [13] and astrophysical plasmas (e.g., geo-cosmic plasmas) $[10,11,28]$. These figures and the shape of surfaces show that exist a noticeable difference between temperature dependence of the mean thermal photoionization cross-section for different species. Figure $2 \mathrm{a}, \mathrm{b}$ show that in the cases of $\mathrm{LiH}^{+}, \mathrm{NaH}^{+}, \sigma(\lambda, T)$ in the region $110 \mathrm{~nm} \leq \lambda \leq 160 \mathrm{~nm}$ significantly increases and becomes noticeable, while decreases for $\lambda$ outside this region. One can notice that the cross-section sudden increase at lower temperature for these cases. Opposite behavior are presented in Figure 3a,b. These figures show that in the cases of $\mathrm{Li}_{2}^{+}$and $\mathrm{LiNa}^{+}, \sigma(\lambda, T)$ significantly increases in the region $400 \mathrm{~nm} \leq \lambda \leq 700 \mathrm{~nm}$ and becomes noticeably and decreasing for $\lambda \geq 700 \mathrm{~nm}$. Also, the temperature dependance is complex.

The obvious qualitative difference in the photodissociation cross-section behavior shown in Figures 2 and 3 can be explained by the fact that they refer to two cases sharply separated at the beginning: Figure 2 refers to highly non-symmetric processes of photodissociation of $\mathrm{HLi}^{+}$and $\mathrm{HNa}^{+}$, while Figure 3 refers to the symmetric case of photodissociation of $\mathrm{Li}_{2}^{+}, \mathrm{Na}_{2}^{+}$and $\mathrm{LiNa}^{+}$.

\subsection{Rate Coefficients}

The behavior of molecular-ion photodissociation rate coefficient $K(\lambda, T)$, calculated by Equation (6), is graphically presented in Figure 4 . In order to enable wider use of these results in various stellar models $[28,29]$, examples of the $\mathrm{NaH}^{+}$and $\mathrm{LiH}^{+}$for temperature range $500 \mathrm{~K} \leq T \leq 20,000 \mathrm{~K}$ and for different wavelengths are studied. The data are presented in Supplementary Material by tables for all species. This allows direct calculation of the spectral absorption coefficients during the process of applying an atmosphere model with the given parameters and composition of plasma. 

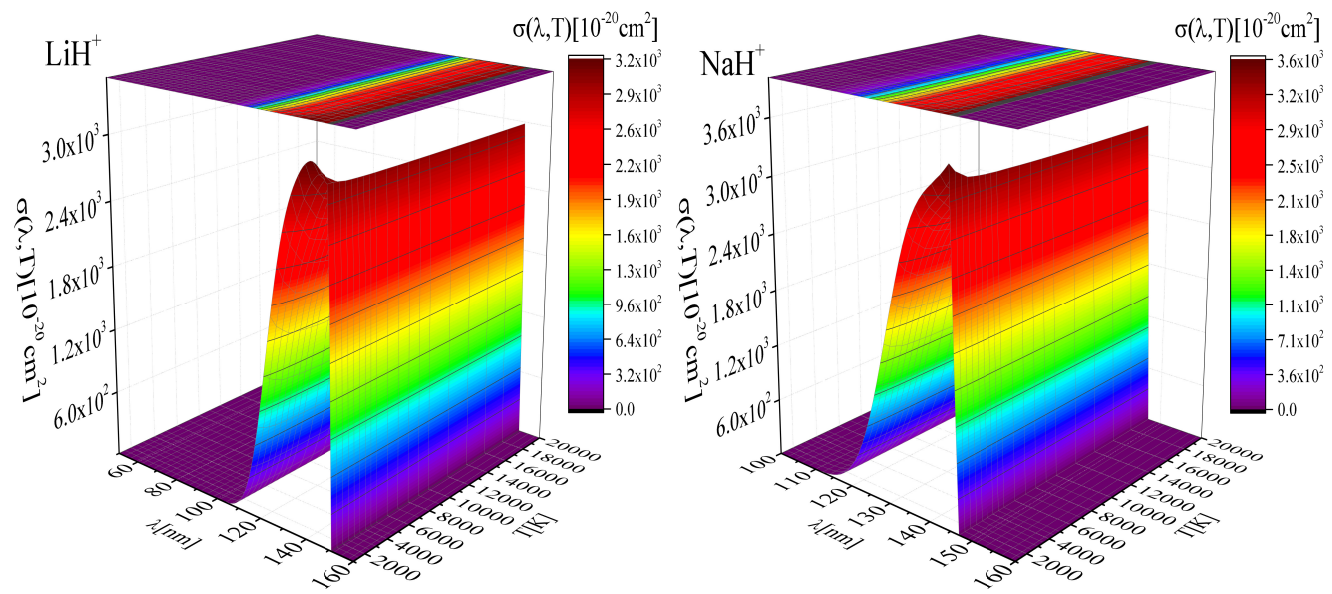

Figure 2. Left: A surface plot of the averaged cross-section $\sigma(\lambda, T)$ for photodissociation of the $\mathrm{LiH}^{+}$ molecular-ion, as a function of $\lambda$ and T; Right: A surface plot of the averaged cross-section $\sigma(\lambda, T)$ for photodissociation of the $\mathrm{NaH}^{+}$molecular-ion, as a function of $\lambda$ and $T$.
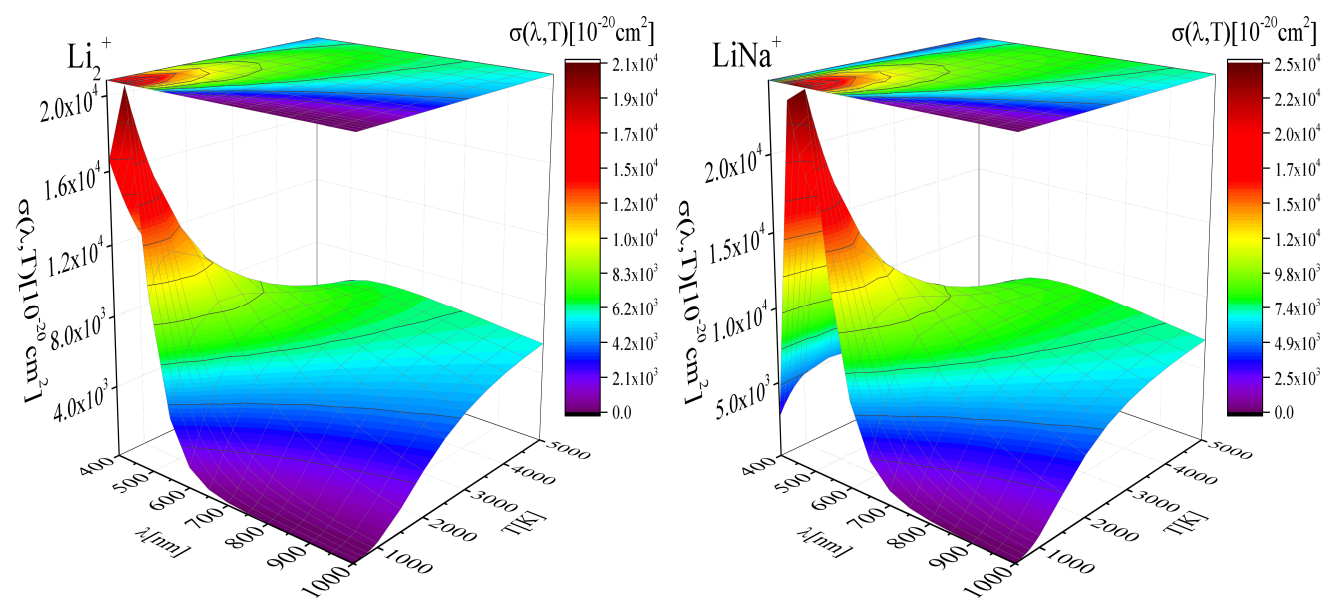

Figure 3. Left: A surface plot of the averaged cross-section $\sigma(\lambda, T)$ for photodissociation of the $\mathrm{Li}_{2}^{+}$ molecular-ion, as a function of $\lambda$ and T; Right: A surface plot of the averaged cross-section $\sigma(\lambda, T)$ for photodissociation of the $\mathrm{LiNa}^{+}$molecular-ion, as a function of $\lambda$ and $T$.

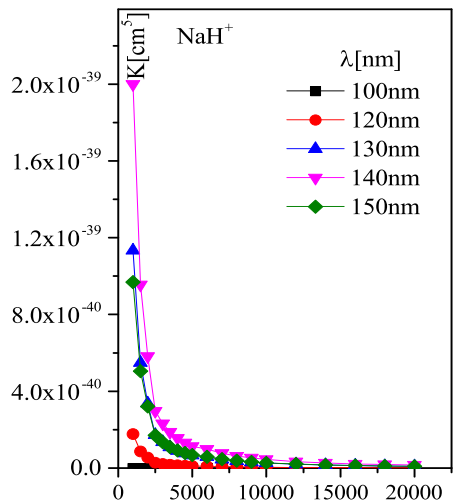

$\mathrm{T}[\mathrm{K}]$

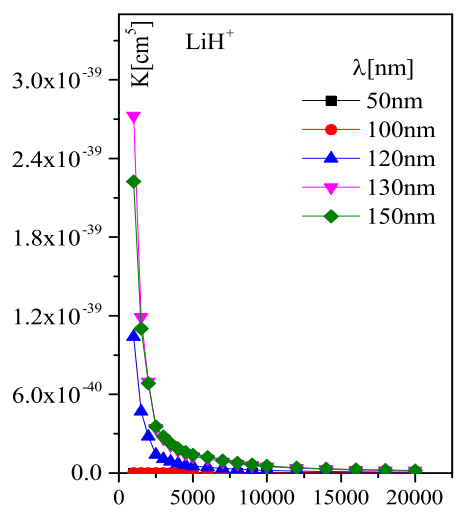

$\mathrm{T}[\mathrm{K}]$

Figure 4. The plot of the spectral rate coefficients $K(\lambda, T)$ for photodissociation of the $\mathrm{NaH}^{+}, \mathrm{LiH}^{+}$, as a function of $T$ and $\lambda$. 
To prepare easier and more satisfying usage of calculated data in modeling as well as in explanation of experimental results in laboratories, we give a simple fitting formula for the photodissociation rate coefficients as a second-degree polynomial (logarithmic) based on a least-square method:

$$
\log (K(\lambda ; T))=\sum_{i=0}^{2} c_{i}(\lambda)(\log (T))^{i}
$$

In Table 1 are listed the coefficients $c_{i}(\lambda)$ i.e., selected fits for the photodissociation rate coefficients. The fitting formula is valid within the temperature range $500 \mathrm{~K} \leq T \leq 5000 \mathrm{~K}$ and wavelengths $400 \mathrm{~nm} \leq \lambda \leq 1000 \mathrm{~nm}$.

Table 1. Data of the selected fits of the Equation (3) to the photodissociation rate coefficient for $\mathrm{Li}_{2}^{+}, \mathrm{Na}_{2}^{+}$and $\mathrm{LiNa}^{+}$.

\begin{tabular}{|c|c|c|c|c|c|c|c|c|c|}
\hline \multirow[b]{2}{*}{$\lambda[\mathrm{nm}]$} & \multicolumn{3}{|c|}{$\mathrm{Li}_{2}^{+}$} & \multicolumn{3}{|c|}{$\mathrm{Na}_{2}^{+}$} & \multicolumn{3}{|c|}{$\mathrm{LiNa}^{+}$} \\
\hline & $c_{0}$ & $c_{1}$ & $c_{2}$ & $c_{0}$ & $c_{1}$ & $c_{2}$ & $c_{0}$ & $c_{1}$ & $c_{2}$ \\
\hline 400 & 115.794 & -83.441 & 11.3946 & 41.7324 & -43.2074 & 5.88338 & 75.7089 & -61.4123 & 8.36583 \\
\hline 500 & 110.628 & -80.5359 & 10.9971 & 82.4755 & -65.1067 & 8.8713 & 85.8781 & -66.8371 & 9.10941 \\
\hline 600 & 97.0323 & -73.0543 & 9.96852 & 80.6419 & -64.0402 & 8.72452 & 76.0451 & -61.3987 & 8.36012 \\
\hline 700 & 84.114 & -65.944 & 8.98853 & 72.9253 & -59.7686 & 8.13487 & 64.6813 & -55.126 & 7.49352 \\
\hline 800 & 72.9344 & -59.7847 & 8.13795 & 64.6227 & -55.1811 & 7.50068 & 54.2741 & -49.373 & 6.69625 \\
\hline 900 & 63.4083 & -54.5306 & 7.41114 & 56.8894 & -50.9076 & 6.90904 & 45.2003 & -44.3536 & 5.99965 \\
\hline 1000 & 55.2672 & -50.0354 & 6.7884 & 49.9549 & -47.0722 & 6.37719 & 37.3453 & -40.0034 & 5.39484 \\
\hline
\end{tabular}

Also, we give table in online material with the data of the selected fits of the Equation (3) to the photodissociation rate coefficient for $\mathrm{LiH}^{+}, \mathrm{NaH}^{+}$.

\subsection{Modeling}

The calculated rate coefficient (see Table 1 and Supplementary material) for the presented plasma parameters could be of interest for the models of Io's atmosphere [30]. This was poorly investigated in this sense till now and consequently, such processes may become important and could be used for better numerical simulations and modeling. In Figure 5 (lower panel) the temperature and density altitude profiles for low density, moderate density and high density of Io modeled atmospheres [30] are presented. The upper panels of Figure 5 shows the photodissociation rate coefficients $K(\lambda, T)$ on the example of $\mathrm{Na}_{2}^{+}, \mathrm{Li}_{2}^{+}$and $\mathrm{LiNa}^{+}$for modeled atmospheres of Io [30,31]. One can notice the growth of coefficients at lower altitudes. Additionally, one can see that the rate coefficients are the largest around $600 \mathrm{~nm}$ for $\mathrm{Na}_{2}^{+}$while for $\mathrm{LiNa}^{+}$at $500 \mathrm{~nm}$ and at $400 \mathrm{~nm}$ for $\mathrm{Li}_{2}^{+}$.

The additional examples i.e., the figures for the moderate-density and high-density models of Io's atmosphere can be found in Supplementary material. We can note that the investigation of these processes is needed for better understanding of the interaction between the Io's atmosphere and Jovian magnetosphere [32] and of the processes in the Jovian environment [33]. Even though lithium has not been spectroscopically discovered at Io till now, some scientists expect its presence [34], and for the estimation of the abundances of lithium as well as of other candidate elements such as fluorine, rubidium, caesium, etc. at Io, the corresponding spectral coefficients are needed. Also, the data for lithium could be of interest in modeling of cold stars with low effective temperatures and enormous high Li abundances (e.g., subgiant J0741+2132, see [35]). 


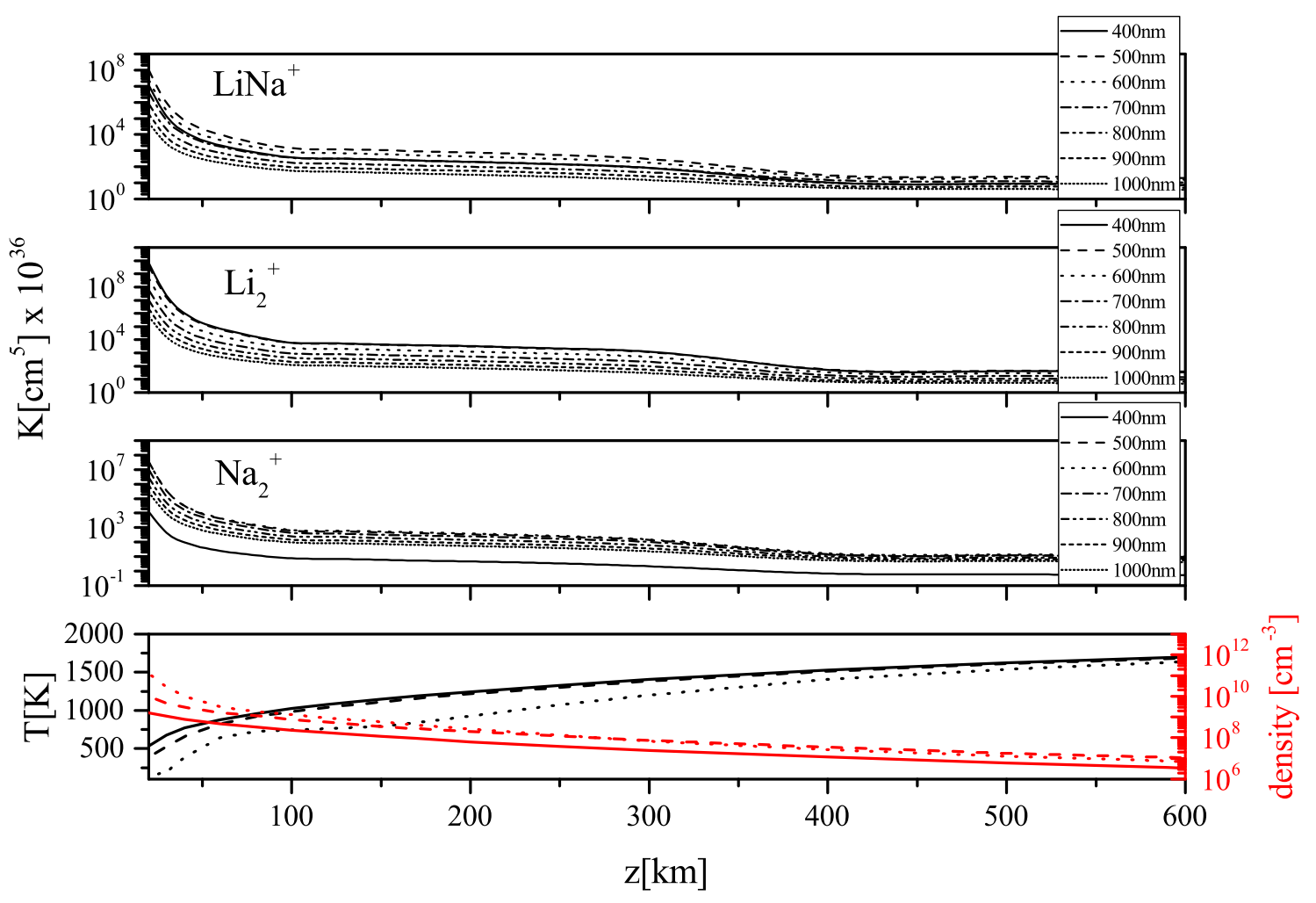

Figure 5. Lower panel: temperature and density altitude profiles for low-density (solid line), moderate-density (dashed) and high-density (dotted line) models of Io's atmosphere. Upper panels: the total photodissociation rate coefficient for $\mathrm{Na}_{2}^{+}$, $\mathrm{Li}_{2}^{+}$and $\mathrm{LiNa}^{+}$for an example of the low-density model of Io's atmosphere [30].

\subsection{Further Usage}

We prepared data in Virtual Observatory (VO) (https:/ / www.ivoa.net/documents) compatible format [36-38] which enable further implementation of results in the A \& M databases like $[27,39]$ thus providing a single location where users can access atomic and molecular data. Also, in order to prepare the easier use of data in practice, we found and give for the rate coefficients a simple and accurate fitting formula as a second-degree polynomial fit to numerical results.

\subsection{Development}

The next step of development is to modify and adapt presented method to enable theoretical research of photodestruction processes which involves larger and complicated molecules for further exploration and explanation of experimentally obtained results e.g., in synchrotron facility (see research of [40-42]) for potential industry, biological, astrobiological and astrochemistry applications [43,44].

\section{The Method and Calculated Quantities}

The rate coefficients and cross-sections are acquired using a quantum mechanical method where the photodissociation process is treated as outcome of radiative transitions between the ground and the first excited adiabatic electronic state of the molecular-ion (see e.g., [45]). The transitions are the result of the interaction of the ion-atom systems electronic component with the electromagnetic field in the dipole approximation. 


\subsection{Photodissociation Cross-Sections}

In accordance with the dipole approximation the partial cross-sections $\sigma_{J, v}(\lambda)$ for the rovibrational states with specified quantum numbers $J$ and $v$, are given in accordance with $[46,47]$ by expression

$$
\begin{array}{r}
\sigma_{J, v}(\lambda)=\frac{8 \pi^{3}}{3 \lambda}\left[\frac{J+1}{2 J+1}\left|D_{J, v ; J+1, E_{i m p}^{\prime}}\right|^{2}\right. \\
\left.+\frac{J}{2 J+1}\left|D_{J, v ; J-1, E_{i m p}^{\prime}}\right|^{2}\right],
\end{array}
$$

where $D_{J, v ; J \pm 1, E_{i m p}^{\prime}}=\left\langle i n, J, v ; R\left|D_{\text {in, fin }}(R)\right|\right.$ fin $, J \pm 1, E^{\prime}>$. $D_{i n ; f i n}(R)$ is the electronic dipole matrix element $D_{\text {in; fin }}(R)=<i n ; R|\mathbf{D}(R)|$ fin; $R>$, where $\mathbf{D}$ is the operator of the dipole moment of the considered system. Here $E^{\prime}=\epsilon_{J, v}+\varepsilon_{\lambda}, E_{i m p}^{\prime}$ and $E^{\prime}, D_{i n, f i n}(R)$ and the states are given in [45]. Figure 6 present illustrative example for $\sigma_{J, v}(\lambda)$ defined by Equation (4) for the case $J=0$ and $v=10$, in the wavelength region $50 \mathrm{~nm} \leq \lambda \leq 1500 \mathrm{~nm}$ for hydrogen diatomic molecular-ion (see [47]).

In the general case mean thermal photodissociation cross-section can be expressed as in $[46,48]$ by the relations

$$
\sigma(\lambda, T)=\frac{\sum_{J, v}(2 J+1) e^{\frac{-E_{I, v}}{k T}} \cdot \sigma_{J, v}(\lambda)}{\sum_{J, v}(2 J+1) e^{\frac{-E_{J, v}}{k T}}},
$$

where $E_{J, v}$ designate the energies of states with the respect to the ground rovibrational states. In Equation (5) $E_{J, v}=E_{\text {dest }}+\varepsilon_{J, v}$, where $E_{\text {dest }}$ is the dissociative energy of the molecular-ion, and the energies $\varepsilon_{J, v}<0$ are defined as in [45,47].

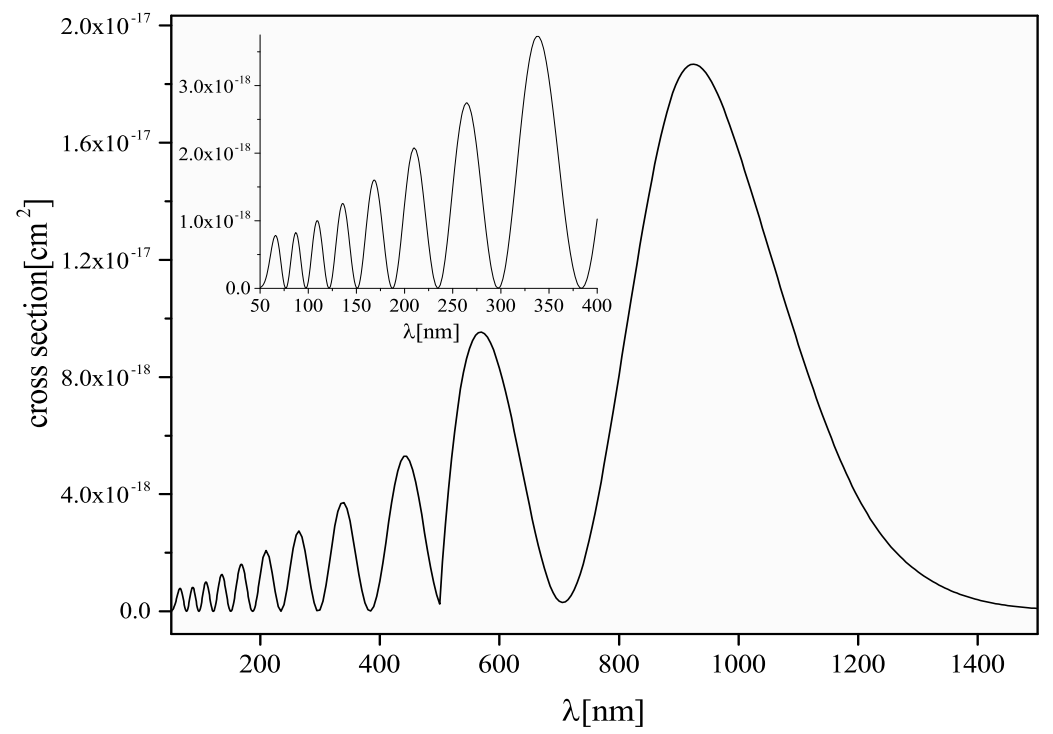

Figure 6. The behavior of the cross-section $\sigma_{J, v}(\lambda)$ Equation (4) for $J=0$ and $v=10$, as a function of $\lambda$ (see [47]).

\subsection{Photodissociation Spectral Rate Coefficient}

Using Equation (5) the photodissociation spectral rate coefficient can be determined as in [46] by the expression

$$
K(\lambda, T)=\sigma(\lambda, T) \cdot \zeta^{-1}(T)
$$


where the factor $\zeta(T)=N(X) N\left(M^{+}\right) / N\left(M X^{+}\right)$in Equation (6) is given by the relation

$$
\zeta(T)=\frac{g_{1} g_{2}}{g_{12}}\left(\frac{\mu k T}{2 \pi \hbar^{2}}\right)^{\frac{3}{2}} \cdot \frac{1}{\sum_{J, v}(2 J+1) e^{\frac{E_{\text {dest }}-E_{J, v}}{k T}}} .
$$

Here $g_{12}, g_{1}$ and $g_{2}$ are the electronic statistical weights of the species, $M X^{+}, X$ and $M^{+}$respectively, and $\sigma(\lambda, T)$ is given by Equation (5). $N(X), N\left(M^{+}\right)$and $N\left(M X^{+}\right)$are density of the $X, M^{+}$and $M X^{+}$, respectively.

This enables further calculation of the spectral absorption coefficients during the process of applying an atmosphere model with the given plasma parameters or e.g., plasma composition in laboratory investigation. The bound-free absorption process (1) i.e., processes of diatomic molecular-ion photodissociation can be characterized by partial spectral absorption coefficients $\kappa(\lambda)$ (see e.g., [26]). It can be presented by relation $\kappa(\lambda)=\sigma(\lambda, T) N\left(M_{2}^{+}\right)=K(\lambda, T) N(M) N\left(M^{+}\right)$where $N\left(M_{2}^{+}\right)$is density of the $M_{2}^{+}$and $\sigma(\lambda, T)$ is average cross-section for photodissociation of this molecular-ion Equation (5) and the coefficient $K(\lambda, T)$ defined by Equation (6). Correspondingly, with the definition of the absorption coefficient, the coefficient $K(\lambda, T)$ is given in units $\mathrm{cm}^{5}$. For the non-symmetric case (2) i.e., $M X^{+}$the relations are similar.

\section{Conclusions}

We have calculated the average cross-section for the photodissociation processes and the corresponding spectral rate coefficient of the diatomic molecular ions $\mathrm{LiH}^{+}, \mathrm{NaH}^{+}$, $\mathrm{Li}_{2}^{+}, \mathrm{Na}_{2}^{+}$and $\mathrm{LiNa}^{+}$for overall range of $500 \mathrm{~K} \leq T \leq 20000 \mathrm{~K}$ and $60 \mathrm{~nm} \leq \lambda \leq 1000 \mathrm{~nm}$. We present the rate coefficients of the corresponding processes in tabulated form, which is appropriate for further use. The obtained results have potential astrophysical use in the improvement of chemistry and modeling of different layers of weakly ionized plasmas e.g., in the atmospheres of various stars and have also been applied to models of the atmosphere of the Io. The results are also important in theoretical and laboratory spectroscopic plasmas research, industry, and technology application.

Supplementary Materials: Supplementary data are available online. Tables presents data for average photodissociation cross-sections and photodissociation rate coefficients for investigated species, fit data, and additional figures present data for potential energy curves for molecules and Io's moderatedensity and high-density models.

Author Contributions: Conceptualization, V.A.S.; Formal analysis, M.S.D., L.M.I. and V.A.S.; Validation, V.A.S.; Visualization, V.A.S.; Writing-original draft, V.A.S. and L.M.I.; Writing-review \& editing, V.A.S. and M.S.D. All authors contributed equally to this work. All authors have read and agreed to the published version of the manuscript.

Funding: This work was funded by the Institute of Physics Belgrade and Astronomical Observatory Belgrade, through the grant by the Ministry of Education and Science of the Republic of Serbia. Also, this research is supported by the European Cooperation in Science and Technology (COST) Action CA18212-Molecular Dynamics in the GAS phase.

Acknowledgments: The authors wish to thank to Magdalena Christova for a fruitful discussion and for the shown attention to this work.

Conflicts of Interest: The authors declare no conflict of interest. The funders had no role in the design of the study; in the collection, analyses, or interpretation of data; in the writing of the manuscript, or in the decision to publish the results.

\section{References}

1. Jraij, A.; Allouche, A.R.; Magnier, S.; Aubert-Frécon, M. Theoretical spin-orbit structure of the alkali dimer cation $\mathrm{K}_{2}{ }^{+}$. Can. J. Phys. 2008, 86, 1409-1415. [CrossRef]

2. Rabli, D.; Mccarroll, R. The low-lying adiabatic states of the $\mathrm{K}_{2}{ }^{+}$alkali dimer. Chem. Phys. Lett. 2019, 723, 82-88. [CrossRef] 


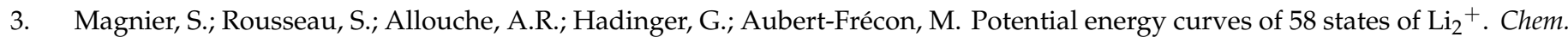
Phys. 1999, 246, 57-64. [CrossRef]

4. Jasik, P.; Wilczyński, J.; Sienkiewicz, J.E. Calculation of adiabatic potentials of $\mathrm{Li}_{2}{ }^{+}$. Eur. Phys. J. ST 2007, 144, 85-91. [CrossRef]

5. Ignjatović, L.M.; Mihajlov, A.A. UV emission/absorption in $\mathrm{H}+\mathrm{A}^{+}$and $\mathrm{H}^{+}+\mathrm{A}$ ion-atom collisions. 19th Summer Sch. Int. Symp. Phys. Ioniz. Gases Contrib. Pap. 1998, 1, 163-166.

6. Ignjatović, L.M.; Mihajlov, A.A. Rate coefficient for the chemi-ionization in slow $\mathrm{Li}^{*}(\mathrm{n})+\mathrm{Li}$ and $\mathrm{Na}$ (n)+Na collisions. Phys. Rev. A 2005, 72, 022715. [CrossRef]

7. Ignjatović, L.M.; Mihajlov, A.A.; Klyucharev, A.N. The rate coefficients of the chemi-ionization processes in slow $\mathrm{Li}^{*}(\mathrm{n})+\mathrm{Na}$ collisions. J. Phys. B 2008, 41, 025203. [CrossRef]

8. Mihajlov, A.A.; Srećković, V.A.; Ignjatović, L.M.; Klyucharev, A.N. The Chemi-Ionization Processes in Slow Collisions of Rydberg Atoms with Ground State Atoms: Mechanism and Applications. J. Clust. Sci. 2012, 23, 47-75. [CrossRef]

9. Efimov, D.K.; Bruvelis, M.; Bezuglov, N.N.; Dimitrijević, M.S.; Klyucharev, A.N.; Srećković, V.A.; Gnedin, Y.N.; Fuso, F. Nonlinear Spectroscopy of Alkali Atoms in Cold Medium of Astrophysical Relevance. Atoms 2017, 5, 50. [CrossRef]

10. Ignjatović, L.M.; Srećković, V.; Dimitrijević, M. Photoionization of the alkali molecular ions in geo-cosmical plasmas. Contrib. Astron. Obs. Skaln. Pleso 2020, 50, 187-192. [CrossRef]

11. Klyucharev, A.N.; Bezuglov, N.N.; Matveev, A.A.; Mihajlov, A.A.; Ignjatović, L.M.; Dimitrijević, M.S. Rate coefficients for the chemi-ionization processes in sodium- and other alkali-metal geocosmical plasmas. New Astr. Rev. 2007, 51, 547-562. [CrossRef]

12. Rai, A.K.; Pati, J.K.; Parigger, C.G.; Dubey, S.; Rai, A.K.; Bhagabaty, B.; Mazumdar, A.C.; Duorah, K. The Plasma Spectroscopic Study of Dergaon Meteorite, India. Molecules 2020, 25, 984. [CrossRef]

13. Beuc, R.; Peach, G.; Movre, M.; Horvatić, B. Lithium, sodium and potassium resonance lines pressure broadened by helium atoms. Astron. Astrophys. Trans. 2018, 30, 315-322.

14. Sharp, C.M.; Burrows, A. Atomic and molecular opacities for brown dwarf and giant planet atmospheres. Astrophys. J. Suppl. Ser. 2007, 168, 140. [CrossRef]

15. Bezuglov, N.; Klucharev, A.; Taratin, B.; Stacewicz, T.; Molisch, A.; Fuso, F.; Allegrini, M. Radiation trapping in an alkali-vapornoble-gas mixture excited by a strong laser pulse. Opt. Commun. 1995, 120, 249-256. [CrossRef]

16. Levine, J.S. The photochemistry of the early atmosphere. In The Photochemistry of Atmospheres; Academic Press: Orlando, FL, USA, 1985; pp. 3-38.

17. Watanabe, A.; Dutta, C.; Nordlander, P.; Kimura, M.; Dalgarno, A. Charge-transfer cross sections for radiative charge transfer in $\mathrm{Na}+\mathrm{H}^{+}$and $\mathrm{K}+\mathrm{H}^{+}$collisions at very low energies. Phys. Rev. A 2002, 66, 044701. [CrossRef]

18. Stwalley, W.C.; Zemke, W.T. Spectroscopy and structure of the Lithium Hydride diatomic molecules and ions. J. Phys. Chem. Ref. Data 1993, 22, 87-112. [CrossRef]

19. Yan, L.; Qu, Y.; Liu, C.; Wang, J.; Buenker, R.J. Ab initio many-electron study for the low-lying states of the alkali hydride cations in the adiabatic representation. J. Chem. Phys. 2012, 136, 124304.

20. Beuc, R.; Movre, M.; Horvatić, B. Time-efficient numerical simulation of diatomic molecular spectra. Eur. Phys. J. D 2014, 68, 59. [CrossRef]

21. Parigger, C.G.; Helstern, C.M.; Jordan, B.S.; Surmick, D.M.; Splinter, R. Laser-Plasma Spatiotemporal Cyanide Spectroscopy and Applications. Molecules 2020, 25, 615. [CrossRef]

22. Parigger, C.G.; Helstern, C.M.; Jordan, B.S.; Surmick, D.M.; Splinter, R. Laser-Plasma Spectroscopy of Hydroxyl with Applications. Molecules 2020, 25, 988. [CrossRef] [PubMed]

23. Beuc, R.; Pichler, G. High-Temperature Optical Spectra of Diatomic Molecules: Influence of the Avoided Level Crossing. Atoms 2020, 8, 28. [CrossRef]

24. Pichler, G.; Beuc, R.; Kokaj, J.; Sarkisyan, D.; Jose, N.; Mathew, J. Photoionization of KCs Molecule: Origin of the Structured Continuum? Atoms 2020, 8, 24. [CrossRef]

25. Pichler, G.; Makdisi, Y.; Kokaj, J.; Mathew, J.; Rakić, M.; Beuc, R. Superheating effects in line broadening of dense alkali vapors. J. Phys. Conf. Ser. 2017, 810, 012013. [CrossRef]

26. Ignjatović, L.M.; Mihajlov, A.A.; Srećković, V.A.; Dimitrijević, M.S. Absorption non-symmetric ion-atom processes in helium-rich white dwarf atmospheres. Mon. Not. R. Astron. Soc. 2014, 439, 2342-2350. [CrossRef]

27. Albert, D.; Antony, B.K.; Ba, Y.A.; Babikov, Y.L.; Bollard, P.; Boudon, V.; Delahaye, F.; Del Zanna, G.; Dimitrijević, M.S.; Drouin, B.J.; et al. A decade with VAMDC: Results and ambitions. Atoms 2020, 8, 76. [CrossRef]

28. Fontenla, J.M.; Avrett, E.; Thuillier, G.; Harder, J. Semiempirical Models of the Solar Atmosphere. I. The Quiet- and Active Sun Photosphere at Moderate Resolution. Astrophys. J. 2006, 639, 441-458. [CrossRef]

29. Hauschildt, P.; Baron, E. Cool stellar atmospheres with PHOENIX. Mem. Della Soc. Astron. Ital. Suppl. 2005, 7, 140.

30. Strobel, D.F.; Zhu, X.; Summers, M.E. On the vertical thermal structure of Io's atmosphere. Icarus 1994, 111, 18-30. [CrossRef]

31. Moses, J.I.; Zolotov, M.Y.; Fegley, B., Jr. Photochemistry of a volcanically driven atmosphere on Io: Sulfur and oxygen species from a Pele-type eruption. Icarus 2002, 156, 76-106. [CrossRef]

32. Wilson, J.K.; Mendillo, M.; Baumgardner, J.; Schneider, N.M.; Trauger, J.T.; Flynn, B. The dual sources of Io's sodium clouds. Icarus 2002, 157, 476-489. [CrossRef]

33. Mendillo, M.; Baumgardner, J.; Flynn, B.; Hughes, W.J. The extended sodium nebula of Jupiter. Nature 1990, 348, 312-314. [CrossRef] 
34. Schaefer, L.; Fegley, B., Jr. Alkali and halogen chemistry in volcanic gases on Io. Icarus 2005, 173, 454-468. [CrossRef]

35. Li, H.; Aoki, W.; Matsuno, T.; Kumar, Y.B.; Shi, J.; Suda, T.; Zhao, G. Enormous Li Enhancement Preceding Red Giant Phases in Low-mass Stars in the Milky Way Halo. Astrophys. J. Lett. 2018, 852, L31.

36. Marinković, B.P.; Vujčić, V.; Sushko, G.; Vudragović, D.; Marinković, D.B.; Đorđević, S.; Ivanović, S.; Nešić, M.; Jevremović, D.; Solov'yov, A.V.; et al. Development of collisional data base for elementary processes of electron scattering by atoms and molecules. Nucl. Instrum. Methods Phys. Res. 2015, 354, 90-95. [CrossRef]

37. Vujčič, V.; Jevremović, D.; Mihajlov, A.A.; Ignjatović, L.M.; Srećković, V.A.; Dimitrijević, M.S.; Malović, M. MOL-D: A Collisional Database and Web Service within the Virtual Atomic and Molecular Data Center. J. Astrophys. Astron. 2015, 36, 693-703. [CrossRef]

38. Jevremović, D.; Dimitrijević, M.; Popović, L.; Dačić, M.; Benišek, V.P.; Bon, E.; Gavrilović, N.; Kovačević, J.; Benišek, V.; Kovačević, A.; et al. The project of Serbian Virtual Observatory and data for stellar atmosphere modeling. New Astron. Rev. 2009, 53, 222-226. [CrossRef]

39. Srećković, V.A.; Jevremović, D.; Vujčić, V.; Ignjatović, L.M.; Milovanović, N.; Erkapić, S.; Dimitrijević, M.S. Mol-D a database and a web service within the Serbian virtual observatory and the virtual atomic and molecular data centre. Proc. Int. Astron. Union 2016, 12, 393-396. [CrossRef]

40. Milosavljević, A.R.; Nicolas, C.; Lemaire, J.; Dehon, C.; Thissen, R.; Bizau, J.M.; Réfrégiers, M.; Nahon, L.; Giuliani, A. Photoionization of a protein isolated in vacuo. Phys. Chem. Chem. Phys. 2011, 13, 15432-15436. [CrossRef]

41. Ranković, M.L.; Cerovski, V.Z.; Canon, F.; Nahon, L.; Giuliani, A.; Milosavljević, A.R. Photodissociation of protonated LeucineEnkephalin peptide in the VUV range. In Proceedings of the 29th International Conference on Photonic, Electronic, and Atomic Collisions (ICPEAC), Deauville, France, 23-30 July 2019; IOP Publishing: Bristol, UK, 2019; Volume 635.

42. Giuliani, A.; Milosavljević, A.R.; Canon, F.; Nahon, L. Contribution of synchrotron radiation to photoactivation studies of biomolecular ions in the gas phase. Mass Spectrom. Rev. 2014, 33, 424-441. [CrossRef]

43. Baiano, C.; Lupi, J.; Tasinato, N.; Puzzarini, C.; Barone, V. The role of state-of-the-art quantum-chemical calculations in astrochemistry: Formation route and spectroscopy of ethanimine as a paradigmatic case. Molecules 2020, 25, 2873. [CrossRef] [PubMed]

44. Singh, S.V.; Vishakantaiah, J.; Meka, J.K.; Sivaprahasam, V.; Chandrasekaran, V.; Thombre, R.; Thiruvenkatam, V.; Mallya, A.; Rajasekhar, B.N.; Muruganantham, M.; et al. Shock Processing of Amino Acids Leading to Complex Structures-Implications to the Origin of Life. Molecules 2020, 25, 5634. [CrossRef] [PubMed]

45. Srećković, V.A.; Mihajlov, A.A.; Ignjatović, L.M.; Dimitrijević, M.S. Ion-atom radiative processes in the solar atmosphere: Quiet Sun and sunspots. Adv. Space Res. 2014, 54, 1264-1271. [CrossRef]

46. Mihajlov, A.A.; Jevremović, D.; Hauschildt, P.; Dimitrijević, M.S.; Ignjatović, L.M.; Alard, F. Influence of chemi-ionization and chemi-recombination processes on hydrogen line shapes in M dwarfs. Astron. Astrophys. 2007, 471, 671-673. [CrossRef]

47. Srećković, V.A.; Ignjatović, L.M.; Jevremović, D.; Vujčić, V.; Dimitrijević, M.S. Radiative and Collisional Molecular Data and Virtual Laboratory Astrophysics. Atoms 2017, 5, 31. [CrossRef]

48. Mihajlov, A.; Sakan, N.; Srećković, V.; Vitel, Y. Modeling of continuous absorption of electromagnetic radiation in dense partially ionized plasmas. J. Phys. A 2011, 44, 095502. [CrossRef] 